\title{
10 ANOS DA ENGENHARIA FÍSICA NA UNIVERSIDADE FEDERAL DO RIO GRANDE DO SUL: ACOMPANHAMENTO DE EGRESSOS E SUAS PERCEPÇÕES SOBRE O CURSO
}

DOI: 10.37702/2175-957X.COBENGE.2021.3607

\author{
Vitória Lunardi Xavier - vitoria.Ix@hotmail.com \\ Universidade Federal do Rio Grande do Sul \\ Ramiro barcelos 1875 \\ 95036-006 - porto alegre - RS \\ Arthur Pereira Dornelles - arthur_dornelles19@hotmail.com \\ Universidade Federal do Rio Grande do Sul \\ Rua Santo Antônio 618 \\ 90220-010 - Porto Alegre - RS
}

Resumo: Em 2020, a Engenharia Física da Universidade Federal do Rio Grande do Sul celebrou sua 1 a década de atividade, ao mesmo tempo que passa por uma reforma curricular devido às novas Diretrizes Curriculares Nacionais e a Curricularização da Extensão. São 10 anos buscando formar profissionais capazes de atuar na indústria de tecnologia da região, tendo conhecimentos sólidos em microeletrônica, ciência de materiais e matemática e física avançada. Esse estudo teve como objetivo, primeiramente, celebrar o aniversário do curso, mapeando os egressos desses 10 anos e investigando suas atuações profissionais e a satisfação com sua formação. Num segundo momento, busca-se dos egressos uma avaliação sobre os aspectos curriculares do curso, procurando entender como o currículo dessa graduação pode ser modernizado. Essa pesquisa foi feita através de um questionário online, dividido nas seções: dados pessoais, mercado de trabalho, pós-graduação, graduação e avaliação do curso e formação posterior. Alcançamos $72 \%$ dos egressos, que se situam $34 \%$ apenas no mercado, $21 \%$ apenas na academia, $37 \%$ em ambos e $8 \%$ em atividades não relacionadas. $A$ satisfação com o curso é alta e eles destacam a multidisciplinaridade e ênfase em física e matemática avançada como aspectos de destaque desse profissional, também reforçam que o curso deve investir em mais disciplinas de programação. Os resultados buscados com esse estudo visam a obter informações sobre os efeitos dessa graduação, a fim de ampliar a divulgação do curso para o mercado e indústria, criando novas oportunidades, e para interessados na área, aumentando 


\section{COBENCE Evento online

a demanda pela graduação.

Palavras-chave: Engenharia Física, acompanhamento de egressos, mudança curricular, mercado de trabalho. academia 
 \\ 10 ANOS DA ENGENHARIA FÍSICA NA UNIVERSIDADE FEDERAL DO RIO GRANDE DO SUL: ACOMPANHAMENTO DE EGRESSOS E SUAS PERCEPÇÕES SOBRE O CURSO}

\section{INTRODUÇÃO}

A graduação de Engenharia Física na Universidade Federal do Rio Grande do Sul (UFRGS) surgiu em 2009 com a Decisão 280/2009 do Conselho Universitário, numa iniciativa conjunta entre o Instituto de Física e a Escola de Engenharia. Essa formação chegou no Brasil por iniciativa da Universidade Federal de São Carlos (UFSCar), que ofereceu a primeira turma no ano de 2000, pela portaria GR no 767/99. Em 2010, a Engenharia Física já existia em cerca de 2 outras universidades e atualmente há 10 cursos de graduação distribuídos por todas as regiões do país.

Na UFRGS, a primeira turma entrou em 2010 e o curso passou a receber anualmente

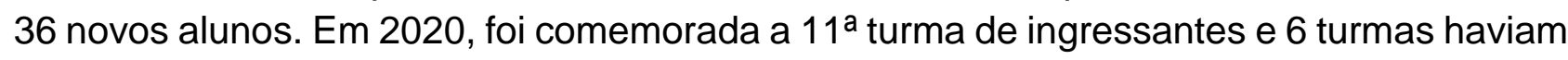
passado pelo tempo mínimo para formação. Segundo a comissão de graduação do curso, até julho de 2020, graduaram-se um total de 53 alunos em Engenharia Física na UFRGS, que saíram com conhecimentos sólidos em microeletrônica, ciência dos materiais, física e matemática avançada. Além disso, a grade curricular também aborda conteúdos como nanomateriais e nanoestruturas, fotônica, tecnologia de vácuo, programação, instrumentação, entre outros.

Por ser um curso recente no país e ter poucos alunos formados, o papel do engenheiro físico na indústria, no mercado e na academia ainda não é amplamente reconhecido. A dúvida sobre a função dessa formação é recorrente entre alunos e professores de outros departamentos e institutos e até os entre próprios estudantes e interessados na área. Essa falta de informação sobre a formação de Engenharia Física também está presente no mercado de trabalho, onde há poucas vagas de estágios, empregos ou trainees em que se especifica "Engenharia Física" como requisito de formação. Além disso, como a UFRGS é a única universidade que oferece o curso na Região Sul do Brasil, esse desconhecimento local do curso tende a ser agravado.

Somado a isso, o cenário atual é de reforma em todos os cursos de engenharia do país, devido às novas Diretrizes Curriculares Nacionais de Engenharia e à Curricularização da Extensão. Portanto, é fundamental que os coordenadores de curso apliquem com eficiência mudanças que modernizem a formação dos alunos. Uma estratégia para isso é coletar avaliações dos egressos, buscando entender em quais aspectos o curso pode se adaptar para atender as demandas da indústria, a partir do seu ponto de vista profissional.

Portanto, primeiramente, este estudo surgiu como maneira de celebrar os 10 anos da criação da Engenharia Física na UFRGS, através do mapeamento dos estudantes que se graduaram neste meio tempo, entendendo onde estão atuando para ter mais informações sobre o impacto dessa formação na indústria e na academia. 
Em um segundo momento, encarando a reforma curricular que será implementada e buscando uma via de melhorar essa tomada de decisão, esse estudo evoluiu para também buscar um retorno sobre como os egressos acreditam que o curso pode melhorar para suprir as necessidades do mercado.

Este estudo, portanto, tem como objetivo mapear os egressos dos primeiros dez anos da Engenharia Física na UFRGS, buscando entender onde estão inseridos dentro do mercado de trabalho e da academia e como acreditam que o curso pode ser modernizado. Espera-se como resultado gerar dados sobre essa formação, buscando intensificar a divulgação do curso no mercado de trabalho, na academia e interessados na área e auxiliar a coordenação do curso na reforma curricular. A consequência a longo prazo deste tipo de estudo é o aumento de oportunidades para quem está na graduação ou concluiu a mesma e aumento de possíveis novos alunos e interessados nessa formação.

\section{MÉTODOS}

Para alcançar os objetivos deste estudo, foi elaborado um formulário online com auxílio de alunos e professores do Instituto de Física da UFRGS. O formulário foi dividido nas seguintes seções:

I) Informações básicas: dados demográficos, ano de ingresso e de formatura; II) Informações profissionais atuais: área de atuação do egresso, onde se subdividia em 2 subseções de acordo com a resposta: a) Mercado de Trabalho: dados sobre o profissional e sobre a organização que está inserido, b) Pós-graduação: dados sobre a pesquisa e a universidade; III) Graduação e avaliação do curso: dados sobre a formação do egresso e avaliação sobre as características curriculares e extracurriculares que regem o curso; IV) Formação posterior: egressos que buscaram outra graduação após finalizar Engenharia Física; V) Encerramento: satisfação do curso, dados e avaliação sobre características que regem o profissional da Engenharia Física.

Foram realizados testes com alguns dos egressos para verificar se havia dificuldade em compreender as perguntas e a dinâmica do questionário e se todos os tópicos necessários para o estudo estavam sendo abordados. No formulário constam tanto perguntas objetivas quanto dissertativas, portanto o estudo ocorreu por análises quantitativas e qualitativas.

O formulário foi elaborado com a ferramenta Google Forms, sendo enviado via email para os egressos que se graduaram até 2020/1, e recebeu respostas por 5 semanas. Os dados obtidos foram tratados e analisados com o software Excel.

\section{RESULTADOS}


Dos 53 egressos, conseguimos contato com 49 deles e obtivemos 38 respostas, o que representa $72 \%$ do público-alvo. A partir das respostas no formulário de pesquisa, adquirimos os dados apresentados nas seguintes seções.

\subsection{Informações básicas}

A idade média dos egressos é 26 anos, sendo o egresso mais novo com 22 anos e o mais velho com 30 anos. Com relação a gênero, 26 (68,4\%) egressos são homens, 11 (29\%) são mulheres e $1(2,6 \%)$ preferiu não dizer. Com relação à cor/raça, $89,5 \%$ se identificam como "Branco", 5,3\% como "Pardo", 2,6\% como "Preto" e 2,6\% como "Sem Declaração" [4].

Em média, os egressos levaram 6,5 anos para se formar, tendo atingido um mínimo de 4,5 anos e máximo de 10 anos. $O$ ano de ingresso e formatura consta na figura abaixo:

Figura 1: Número de Egressos por Ano de Ingresso e Formatura

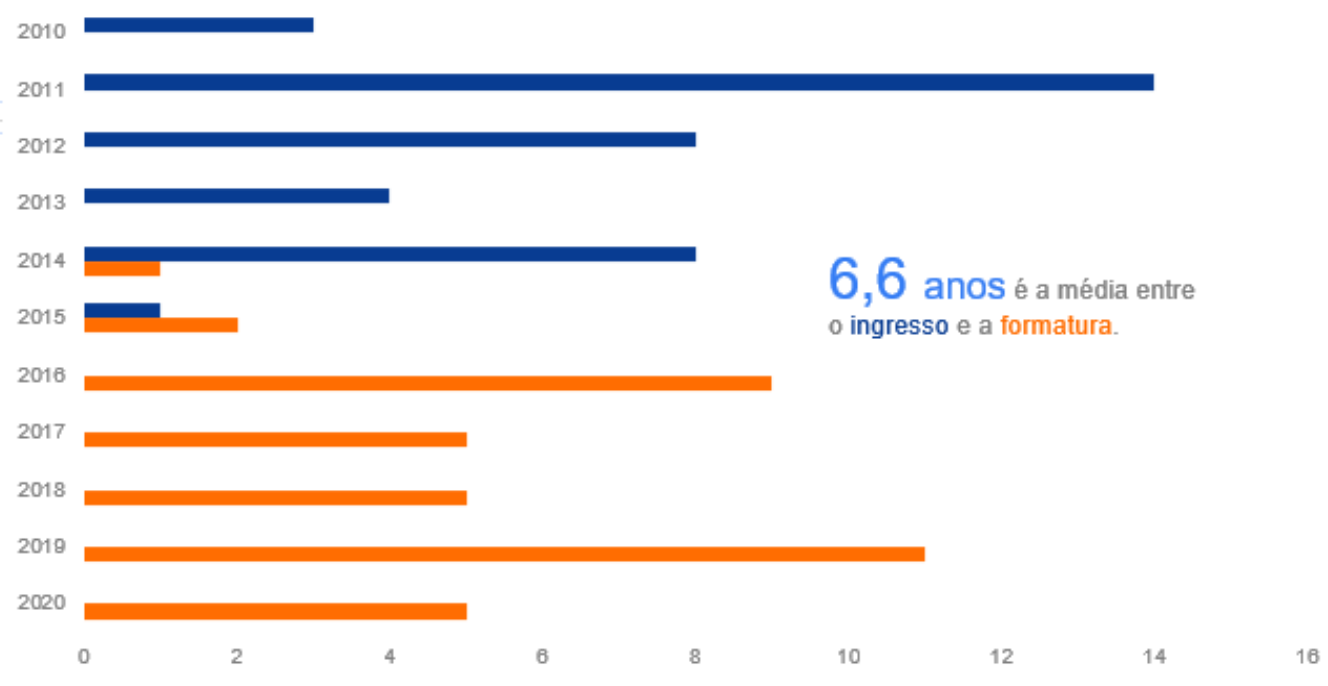

\subsection{Informações Profissionais Atuais}

Há $36,8 \%$ dos egressos concomitantemente no mercado de trabalho e na pósgraduação, 34,2\% apenas no mercado de trabalho, 21\% apenas na pós-graduação, 5,3\% em situação de desemprego e 2,6\% mudou de carreira.

\subsection{Mercado de trabalho}

Setenta e um por cento dos egressos estão no mercado de trabalho e o tipo de organização da atividade profissional varia entre: privada (74\%), empreendimento próprio (11\%), pública $(7 \%)$, economia mista $(4 \%)$, concomitantemente em empresa privada e empreendimento próprio (4\%).

Para investigar a faixa salarial obtivemos apenas 19 respostas, sendo dois deles em moedas diferentes do real brasileiro. A faixa salarial dos que recebem em real brasileiro apresenta uma média de $\mathrm{R} \$ 5.229,00$.

A função de cada egresso no mercado de trabalho e as respectivas empresas podem ser vistas na Tabela 1: 
Tabela 1: Funções e Empresas de Egressos no Mercado de Trabalho.
Função
№ Egressos
Empresas

\begin{tabular}{ccc}
\hline Cientista de dados & 3 & $\begin{array}{c}\text { DLL Group, Openbox.ai, } \\
\text { Plugar Data \& Intelligence }\end{array}$ \\
\hline $\begin{array}{c}\text { Engenheiro de Aprendizado de } \\
\text { Máquina }\end{array}$ & 3 & DNV GL \\
\hline Trainee & 2 & Aprix, Axur \\
\hline Pesquisador & 2 & $\begin{array}{c}\text { NTC ZCUas Quero-Quero } \\
\text { Técnica de Viena }\end{array}$ \\
\hline Consultor & 2 & SAP \\
\hline Engenheiro & 2 & Siemens, UFRGS \\
\hline Desenvolvedor de Hardware & 2 & VanellusRad \\
\hline Chefe de Análise & 1 & Aprix \\
\hline Engenheiro de Telecomunicações & 1 & Infortel \\
\hline Laboratorista & 1 & PUCRS \\
\hline Analista de Controle de Processos & 1 & Sindus Andritz Ltda \\
\hline Analista de Desenvolvimento de \\
Software
\end{tabular}

Sobre satisfação com ocupação, $63 \%$ dos egressos sentem-se muito satisfeitos; $33,3 \%$ sentem-se satisfeitos e $3,7 \%$ sente-se neutro com a satisfação.

Pode-se observar na figura 2 o quão preparados os egressos se sentiram em encarar o mercado de trabalho após a graduação.

Figura 2: Preparação para o Mercado de Trabalho 


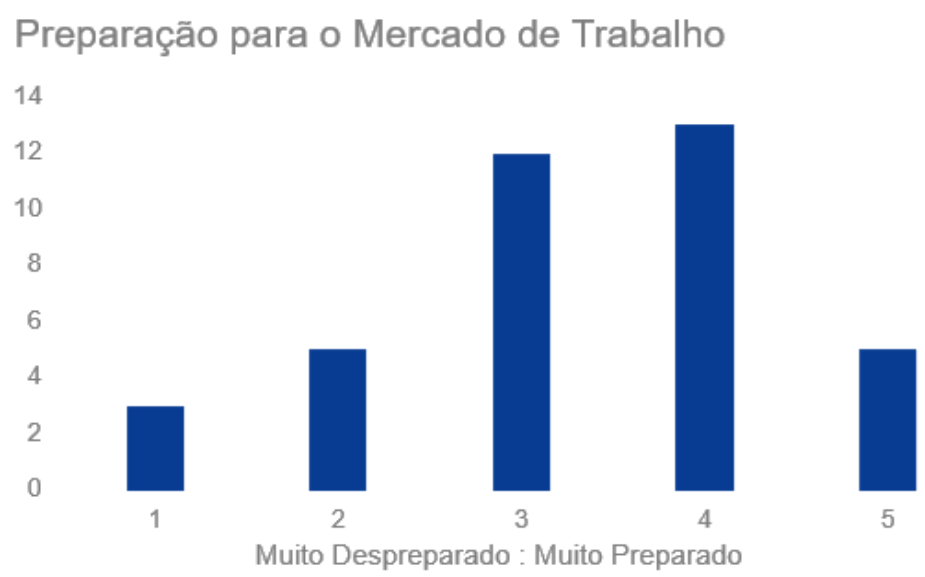

Na Figura 2, percebe-se que a moda dos egressos se localiza entre preparado e muito preparado.

\subsection{Pós-graduação}

Cinquenta e oito por cento dos egressos atualmente está fazendo alguma pósgraduação. Cruzando os dados de tipo de instituição (pública ou privada) com grau da pósgraduação, obtém-se o seguinte gráfico:

Figura 3.: Grau de Pós-Graduação em Curso por Egresso

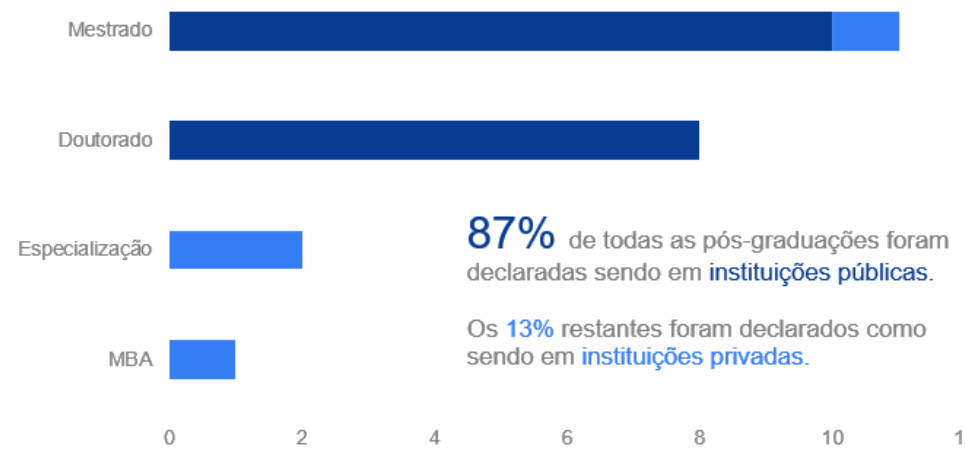

A área acadêmica mais popular, contanto com 8 egressos nela, foi a Microeletrônica, seguida com dois egressos nas áreas de Engenharia dos Materiais, Ciência da Computação, Ciência dos Materiais e Engenharia Mecânica e com um egresso em Análise Empresarial, Ciências Aplicadas, Computação Quântica, Econofísica, Ensino de Física, Física Teórica, Física Experimental, Neurociências e Neuroengenharia. Estão principalmente na Universidade Federal do Rio Grande do Sul (UFRGS, 13 egressos), mas também em ENSICAEN, Universidade Técnica de Viena, Universidade de Grenoble, Pontifícia Universidade Católica do Rio Grande do Sul (PUC-RS), Universidade do Vale do Rio dos Sinos (UNISINOS), Universidade de West Bohemia, ETH Zurich e ISD/Natal. 


\subsection{Graduação e Avaliação do Curso}

Dividimos as atividades acadêmicas da graduação em atividades extracurriculares, estágios, intercâmbio e disciplinas da grade curricular. Os resultados serão elaborados seções seguintes.

\subsection{Atividades Extracurriculares}

As atividades extracurriculares realizadas pelos egressos durante graduação foram iniciação científica $(91 \%)$, diretório acadêmico $(37,8 \%)$, empresa júnior $(18,9 \%)$, monitoria acadêmica (16,2\%), extensão $(7,9 \%)$, atlética $(5,4 \%)$ e nenhuma $(5,4 \%)$.

\subsection{Estágios}

Os alunos fizeram estágios nas empresas CEITEC, Infortel Telecom, DNV-GL, HT Micron, NOVUS Produtos Eletrônicos, Olvebra, DLL Group, TDK Epcos, ENGEP, OpenBox.ai, Drenomaster, SAP, DMAE, Duo Engenharia, L'Oréal, Société Générale de Surveillance, Instituto Brasileiro de Tecnologia do Couro, Calçado e Artefatos, Sociedade do Cálculo Matemático de Paris, CEA - Commissariat à l'Énergie Atomique et aux Energies Alternatives (França), New York-Presbyterian Hospital, Toth LifeCare, MedPlace, PUCRS, Centro de Nanociência e Nanotecnologia (UFRGS), Laboratório de Implantação lônica (UFRGS), Laboratório de Criogenia (UFRGS), Laboratório Nacional de Luz Síncrotron (CNPEM), Warren, NTZ ZCU e Bleistahl Metalúrgica.

\subsection{Intercâmbio}

Sobre intercâmbios realizados durante a graduação para equivalência de estudo, $63,2 \%$ dos egressos não realizaram nenhum, $26,3 \%$ foram pelo programa Ciências sem Fronteiras, 5,3\% para as Écoles Centrales e 2,6\% para ParisTech e outro 2,6\% pela UNIGOU.

\subsection{Disciplinas e avaliação do curso}

Os egressos foram questionados sobre quais disciplinas ou assuntos deveriam ser agregadas a grade do curso, ou seja, quais conhecimentos fizeram falta em suas atividades profissionais. As respostas mais comuns foram:

- Programação: assuntos que abordem introdução à programação, lógica básica, diferentes linguagens de programação e/ou estruturas de dados.

- Ciência de dados: assuntos que abordem análise e visualização de dados, conceitos de Inteligência Artificial, Machine Learning, Big Data e Internet das Coisas.

- Gestão: assuntos que abordem gestão de projetos, de qualidade, de pessoas e/ou de empresas.

Em menor quantidade também surgiram sugestões de agregar: desenho técnico com ferramentas computacionais, mecânica de fluídos, termodinâmica clássica e disciplinas que discutam a integração de sociologia, ciência e tecnologia.

Além de sugestões de assuntos novos na grade curricular do curso, também surgiram propostas de aprimorar ou reforçar assuntos/disciplinas de estatística, eletrônica analógica e instrumentação física. 
Outros comentários que surgiram foram sobre a necessidade de mais disciplinas eletivas, mais disciplinas práticas e possibilidade de ênfase em algum assunto ao longo da graduação.

Também foi questionado quais as disciplinas foram menos importantes para formação de Engenharia Física, portanto poderiam ser retiradas da grade curricular sem prejuízo. Em resposta ao questionário foram indicadas disciplinas superficiais, com sobreposição de conteúdo e distantes da realidade do mercado de trabalho. Detalhes foram encaminhados a coordenação do curso para que considerassem na iminente reformulação curricular para adequação às novas DCNs de Engenharia e à Curricularização da Extensão. Também foi criticada a quantidade excessiva de disciplinas voltadas à ciência dos materiais.

Os egressos apontaram que os maiores diferenciais da Engenharia Física são: multidisciplinaridade, base sólida em matemática e física e complexidade das disciplinas. Alegam que esses fatores do curso resultam num diferencial de capacidade de aprendizado amplo e flexível.

Quando questionados sobre a satisfação do curso, obteve-se o seguinte resultado:

Figura 4: Qual sua satisfação com o curso?

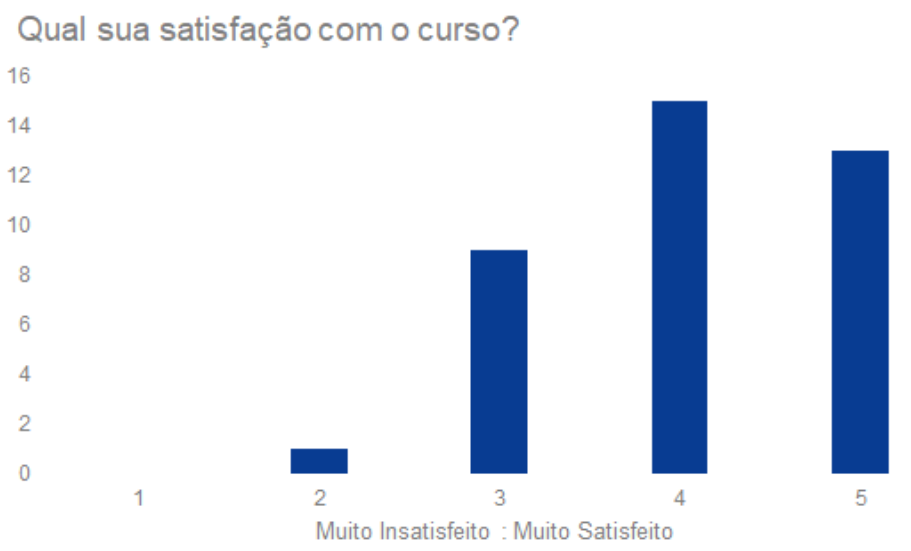

Observa-se pela Figura 4 que o grau de satisfação dos egressos de Engenharia Física da UFRGS é alto.

\subsection{Formação posterior}

Nessa sessão buscamos investigar a incidência de egressos que buscaram uma nova graduação após concluírem a Engenharia Física. Apenas 2 egressos estão cursando outra graduação, que são os cursos de Ciências da Computação e Medicina.

\subsection{Encerramento}

Nas seções Graduação e Avaliação do Curso, Mercado de Trabalho e PósGraduação, questionamos os participantes da pesquisa de que maneira a Engenharia 
Física tinha impactado seu ambiente de trabalho e sua formação. As respostas mais frequentes foram:

- Fornecendo ferramentas e base conteudista: a Engenharia Física forneceu conhecimento e raciocínio físico e matemático avançados para tomadas de decisões e desenvolvimento de soluções. Também proveu ferramentas como programação, experiência em laboratórios e outros, que foram necessárias na atuação profissional;

- multidisciplinaridade do currículo: a grade diversa e sólida forma profissionais generalistas, que têm uma visão ampla do problema a ser resolvido;

- capacidade de aprendizado e adaptação: o aluno de Engenharia Física aprende a aprender devido as disciplinas complexas e diversas do curso;

- forma inovadora de pensar e resolver situações;

- interpretações mais analíticas dos problemas;

Sobre a ausência de título profissional específico (CONFEA) para Engenharia Física, os egressos foram questionados sobre a falta que o registro faz.

Figura 5: Quanto o título profissional específico (CONFEA) fez falta?
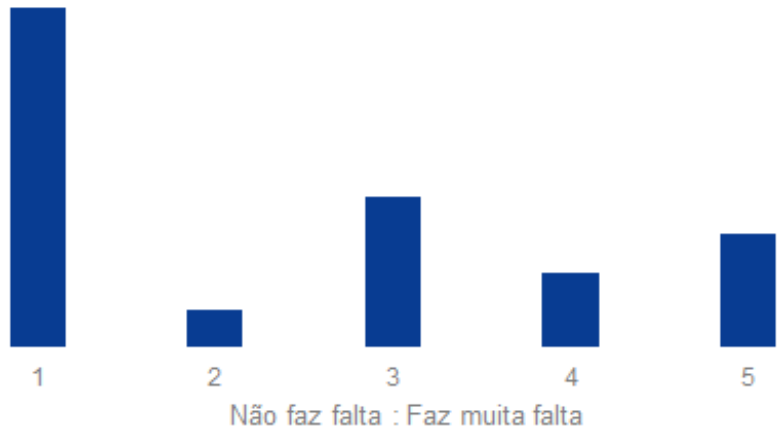

Onde 52,6\% apontaram que o CREA específico para Engenharia Física não faz falta ou faz pouca falta e $26,3 \%$ apontaram fazer falta ou fazer muita falta. Observa-se, portanto, que não há um consenso sobre esse tópico.

\section{DISCUSSÕES E CONCLUSÕES}

Com base nos resultados apresentados acima, percebe-se que o objetivo do estudo foi alcançado, dado que foram coletados dados pessoais, profissionais e educacionais sobre $70 \%$ dos egressos da primeira década da Engenharia Física na UFRGS, além de buscar uma avaliação da estrutura do curso. Com isso, um volume interessante de dados sobre o que a Engenharia Física oferece na graduação, no mercado e na academia foi criado, e com a divulgação desse estudo esperamos que essas informações atinjam novas empresas, possíveis futuros alunos, colegas de outros cursos e outras universidades. Assim, as oportunidades disponíveis para esses alunos tendem a aumentar, bem como a quantidade de estudantes interessados na área. 
A seção de avaliação do curso de fato proveu informações importantes e necessárias para que a coordenação do curso tomasse decisões sobre a reforma curricular.

Por fim, o acompanhamento de egressos de Engenharia Física deve ser um trabalho contínuo. Esperamos que esse estudo motive não apenas outras versões dessa mesma pesquisa na UFRGS, mas também em outras universidades que oferecem o curso, para que a Engenharia Física ganhe cada vez mais visibilidade no mercado de trabalho e no meio acadêmico brasileiro, além de manter o currículo sempre moderno e atualizado.

\section{Agradecimentos}

Agradecemos, primeiramente, aos egressos da Engenharia Física da UFRGS, que "abriram caminho" e estão criando oportunidades para os atuais alunos do curso e receberam esse questionário com muito carinho. Aos professores do Instituto de Física e Escola de Engenharia, com especial atenção ao Cristiano Krug e Carlo Requião, que sempre foram extremamente atenciosos com nossas solicitações e motivaram e ajudaram na construção desse estudo. Por fim, agradecemos aos nossos colegas da Engenharia Física da UFRGS, que sempre estiveram abertos a discussões que visam a melhorar o curso e receberam esse tipo de projeto de braços abertos.

\section{REFERÊNCIAS}

IF - Instituto de Física da UFRGS. PROJETo PEDAGógICo DO CURSO DE ENGENHARIA FÍSICA. 2016. Disponível em: https://www.if.ufrgs.br/if/wpcontent/uploads/2016/12/PPC-Eng-Fis-2016.pdf .

Acesso em: 08 Mai. 2021.

VICINO, Leonardo Minuchelli. MAPEAMENTO DOS EGRESSOS DA ENGENHARIA FÍSICA DA UFSCAR: LEVANTAMENTO DE INFORMAÇÕES PROFISSIONAIS E DE OPINIÕES SOBRE O CURSO..2019. Monografia (Graduação) - Curso de Engenharia Física. Universidade Federal de São Carlos, São Carlos, 2019.

\section{e-MEC. CADASTRO NACIONAL DE CURSOS E INSTITUIÇÕES DE EDUCAÇÃO} SUPERIOR. Disponível em: https://emec.mec.gov.br/emec/nova-index/consulta-avancada . Acesso em: 23 Abr. 2021.

BRASIL- Instituto Brasileiro de Geografia e Estatística. Conheça o Brasil - População: Cor ou Raça. Disponível em: https://educa.ibge.gov.br/jovens/conheca-o-brasil/populacao/18319-cor-ou-raca.html . Acesso em: 20 Jan. 2021. 


\title{
10 YEARS OF ENGINEERING PHYSICS OF FEDERAL UNIVERSITY OF RIO GRANDE DO SUL: TRACKING OF GRADUATES AND THEIR FEEDBACK ABOUT THE COURSE
}

\begin{abstract}
In 2020, the Engineering Physics Program of the Federal University of Rio Grande do Sul (UFRGS) celebrated its tenth anniversary going through strict curriculum change due to the new Brazilian National Curricular Guidelines and Curricularization of University Outreach Programs. For 10 years, the University has been preparing students to work and innovate in the local technology industry, with knowledge in microelectronics, material science, and advanced Mathematics and Physics. Firstly, this research aimed to celebrate the program's anniversary by tracking students that graduated from Engineering Physics of UFRGS, taking note of their career paths, and how satisfied they are with them. Secondly, the graduates gave feedback about the current EP's curriculum based on their experience on the market, which was used to understand how the program can be improved. This research was conducted through an online form, divided into sections: personal information, job market, graduate studies, undergraduate studies and curriculum assessment, and further education. We were able to reach $72 \%$ of the graduates, where $34 \%$ are in the job market, $21 \%$ are academics, $37 \%$ are both and $8 \%$ are non-related activities. They demonstrated a high course satisfaction and highlighted that the multidisciplinary and the emphasis on advanced Mathematics and Physics are unique qualities; they also suggested that the curriculum should have more programming-related courses. The outcome gave us the tools to obtain information about the impact of Engineering Physics. This way we can increase the outreach to the job market and industry and create new opportunities for EP students.
\end{abstract}

Keywords: engineering physics, graduates tracking, curricular change, job market, graduation 